\title{
Successful use of sacral neuromodulation after failed bladder augmentation
}

\author{
Nathan T. Rasmussen, MD; Michael L. Guralnick, MD, FRCSC; R. Corey O'Connor, MD
}

\begin{abstract}
Sacral neuromodulation has become a standard minimally invasive therapy for refractory urinary urge/frequency and urge incontinence. Prior to the widespread use of sacral neuromodulation, augmentation cystoplasty was a standard treatment for refractory overactive bladder $(\mathrm{OAB})$. The use of sacral neuromodulation following bladder augmentation has not been previously reported in the literature. We report 2 cases of successful sacral neuromodulation in patients with $\mathrm{OAB}$ refractory to bladder augmentation.
\end{abstract}

Can Urol Assoc J 2009;3(5):E49-E50

\section{Introduction}

Since the late 1990s, sacral neuromodulation has become a standard minimally invasive therapy in the management algorithm of refractory urinary urgency/frequency and urge incontinence. ${ }^{1}$ We report the first 2 cases of sacral neuromodulation in patients with continued overactive bladder (OAB) symptoms following failed augmentation cystoplasty.

\section{Case 1}

A 46-year-old female was referred to the urology clinic with a 6-year history of urinary urgency, frequency and incontinence. Prior to presentation, oral anticholinergic medications and pelvic floor rehabilitation were unsuccessful. She underwent an ileal bladder augmentation 4 years earlier without change in her symptoms. Upon referral, the patient was voiding every 30 to 60 minutes, with 4 to 5 episodes of urge incontinence per day. Genitourinary physical exam and urine analysis were negative for abnormalities. Postvoid residual urine volume was 0 cc. Cystoscopy revealed a 350 cc awake bladder capacity and a wellconstructed augmented bladder. Voided cytology was negative for pathology. Video urodynamic studies demonstrated hypersensate bladder and detrusor overactivity. Bladder compliance was normal. She underwent cystoscopy with intradetrusor injection of 200 units of type A Botulinum toxin. Her symptoms remained unchanged.

The patient underwent staged sacral neuromodulator

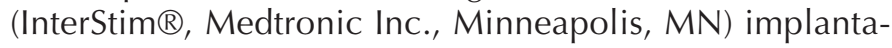
tion in the right third sacral (S3) foramina. Following stage I implantation, the patient began voiding every 3 hours during the day. She denied nocturia or urinary incontinence. The patient proceeded to an uneventful second stage implantation. After 14 months of follow-up, the patient's symptomatic improvement remains stable.

\section{Case 2}

A 57-year-old woman presented to our institution with a history of urinary urgency/frequency and pelvic pain. She had been previously treated with multiple anticholinergic agents, as well as supratrigonal cystectomy with ileocystoplasty without success. Bladder diary revealed voids every 1.5 hours with volumes ranging from $50 \mathrm{cc}$ to $220 \mathrm{cc}$. Genitourinary physical exam and urinalysis were unremarkable. A video urodynamic evaluation demonstrated a hypersensate bladder, adequate compliance and a cystometric capacity of $300 \mathrm{cc}$. The patient's postvoid residual urine volume was nil. Various additional anticholinergic medications were administered to the patient, without improvement in her symptoms.

Subsequently, the patient underwent a staged sacral neuromodulator implantation into the left S3 foramen. Her urinary frequency improved to every 3 to 4 hours with volumes ranging from $300 \mathrm{cc}$ to $500 \mathrm{cc}$. The patient's symptomatic improvement has persisted with a follow-up of 17 months.

\section{Discussion}

Historically, bladder augmentation has been performed as salvage therapy for $\mathrm{OAB}$ patients who are refractory to other more conservative measures. ${ }^{2}$ The detubularized bowel interposition interrupts detrusor contractions and, therefore, detrusor overactivity. ${ }^{3}$ Furthermore, the procedure is believed to provide symptomatic improvement by increasing bladder compliance and dampening increases in intra- 
vesical pressure resulting from uninhibited bladder contractions. The end result is decreased urinary urgency. More recently, sacral neuromodulation and intradetrusor injection of type A Botulinum toxin have emerged as a less invasive therapy for refractory $\mathrm{OAB}$ patients. ${ }^{1,4}$ As a result, the current use of bladder augmentation in this population has drastically decreased.

We present 2 patients that underwent successful sacral neuromodulation after failed enterocystoplasty. The mechanism of action of sacral neuromodulation is hypothesized to involve electrical stimulation of somatic afferents, which modulates voiding and continence reflex pathways in the central nervous system. ${ }^{5}$ It is likely that although the augmentations in our patients provided acceptable bladder capacities, they were insufficient in adequately dissipating the uninhibited detrusor contractions, thus resulting in continued lower urinary tract symptomatology. The addition of sacral neuromodulation in these patients allowed us to improve their symptoms in a minimally invasive fashion and avoid the potential complications of revision enterocystoplasty.

\section{Conclusion}

We report the first cases of failed bladder augmentation successfully treated with pelvic neuromodulation. This therapy may be considered for treatment of similar patients.
From the Department of Urology, Medical College of Wisconsin, Milwaukee, WI

Competing interests: None declared.

This paper has been peer-reviewed.

\section{References}

1. Scheepens WA, Van Koeveringe GA, De Bie RA, et al. Long-term efficacy and safety results of the twostage implantation technique in sacral neuromodulation. BJU Int 2002;90:840-5.

2.. Goodwin WE. Results of ileocystoplasty. J Urol 1958;80:461-6.

3. Dmochowski R. Interventions for detrusor overactivity: the case for multimodal therapy. Rev Urol 2002;4(Suppl4):S19-27.

4. Rapp DE, Lucioni A, Katz EE, et al. Use of botulinum-A toxin for the treatment of refractory overactive bladder symptoms: an initial experience. Urology 2004;63:1071-5.

5. Leng WW, Chancellor MB. How sacral nerve stimulation neuromodulation works. Urol Clin NA 2005;32: 11-8.

Correspondence: Dr. R. Corey O'Connor, Department of Urology, Medical College of Wisconsin, 9200 W. Wisconsin Ave., Milwaukee, WI 53226; fax: 414-805-0771; corey0c@aol.com 